\title{
Number of Lymph Nodes Positive for Tumor by H\&E
}

National Cancer Institute

\section{Source}

National Cancer Institute. Number of Lymph Nodes Positive for Tumor by H\&E. NCI

Thesaurus. Code C157146.

The quantity of lymph nodes that were positive for a neoplastic lesion with hematoxylin and eosin (H\&E) procedures. 